\title{
The Elderly Housing Choice Based on the Improved FAHP
}

\author{
Jia Hongjun ${ }^{1, *}$, Hang Xiaoya ${ }^{2}$ and Sun Lingzhi ${ }^{2}$ \\ ${ }^{I}$ Department of Resources and Civil Engineering, Shandong University of Science and Technology, Taian, Shandong, \\ 271000, P.R. China; ${ }^{2}$ School of Civil Engineering and Architecture, Shandong University of Science and Technology, \\ Qingdao, Shandong, 266590, P.R. China
}

\begin{abstract}
With the intensification of aging, the elderly housing choice has become one of the social problems that has attracted greater public attention. Through scientific and systematic questionnaires and statistical analysis, the main factors affecting the elderly housing choice were concluded, an evaluation index system of elderly housing choice was established, and an effective criteria of calculating the system evaluation index values $Z_{k}$ was formed based on the fitting function of matlab. On this basis, a method of the elderly housing choice was proposed, scientificalness and rationality of this method has been verified in the case study.
\end{abstract}

Keywords: Aging, elderly housing choice, FAHP, judgment value, matlab.

\section{INTRODUCTION}

With the second baby boom population which occurred from the 1960s to the mid-1970s into old age, China's elderly population began to increase at an average annual growth rate of 6.2 million [1]. This large-scale, high-speed growth pattern corresponds to the diversified and blowout development of the elderly consumers' demand. Meanwhile, changes of elderly living preferences, enhancement of purchase desire and the increase of income indicate the inevitable expansion of the elderly housing market demand. Nowadays, for the older age groups, residence is no longer a simple concept of physical space, it has gradually become a kind of social environment not only to meet the personal material demand more to meet the spiritual needs. Through the establishment of precise mathematical model, and a systematic and comprehensive evaluation of alternative sets, some important information about alternative system performance and living environment can be provided to consumers. Then, the decision-making risk of consumers will be reduced effectively.

\section{RESEARCH SIGNIFICANCE}

Unlike welfare houses, old folks' homes and other social welfare facilities, elderly housing is a kind of real estate product bred out by the interaction between consumers, developers and government. Survey data shows that based on practical considerations of children living and their own needs, those elderly who want to stay in elderly housing immediately and other who would like to stay there in the future accounted for $30 \%$ and $63 \%$ of the total number of survey respectively. The transformation of old groups' ideology provides unlimited business opportunities for real estate developers. Construction of elderly housing is imperative.

In addition, the family structure of "four-two-one" and fast-paced social life caused a number of two-income families lack sufficient time to take care of elderly people, which make daily life issues of elderly become increasingly prominent. With the development of the integrated system, ideas of old people have made a qualitative leap, the elderly not only want to obtain the convenience of living more desire spiritual satisfaction from elderly housing [2]. With many uncertainties that affect elderly housing choice, it's difficult for general public to make a scientific and rational judgment or decision. An unripe idea can result in a phenomenon of "selling old to buy new", which may cause losses to consumers. Therefore, the research in this paper will help consumers to make a comprehensive preparation before buying house, and it will provide a favorable reference to solve the pension problem.

\section{MODEL ESTABLISHMENT}

\subsection{Establishment of Evaluation Index System}

A questionnaire was made to explore the main factors which affect the elderly housing choice. 500 questionnaires were issued to elderly and 479 valid questionnaires were recovered. 300 questionnaires were issued to middle-aged group and 283 valid questionnaires were recovered. Excluding the index less than $50 \%$ and retaining the index more than $50 \%, 6$ indicators were concluded. Then the following evaluation system was established.

\subsection{Solution of the Weight Set}

Analytic Hierarchy Process (AHP) is an analytical method of multi-objective decision, which is commonly used to compare the superiority of multiple factors $[3,4]$. The evaluation index system established in this paper includes 
Table 1. Evaluation index system of elderly housing choice.

\begin{tabular}{|c|c|c|}
\hline Target Layer & Criteria Layer & Sub-Criteria Layer \\
\hline \multirow{14}{*}{$\begin{array}{l}\text { Evaluation index system of } \\
\text { elderly housing choice } U\end{array}$} & Housing price $\mathrm{U}_{1}$ & Null \\
\hline & \multirow{2}{*}{ Traffic $\mathrm{U}_{2}$} & Public transport distance $U_{21}$ \\
\hline & & The number of public bus $U_{22}$ \\
\hline & \multirow{2}{*}{ Leisure and entertainment $U_{3}$} & Distance $\mathrm{U}_{31}$ \\
\hline & & Number $\mathrm{U}_{32}$ \\
\hline & \multirow{2}{*}{ Large shopping centers $U_{4}$} & Distance $\mathrm{U}_{41}$ \\
\hline & & Number $\mathrm{U}_{42}$ \\
\hline & \multirow{2}{*}{ Comprehensive hospital $U_{5}$} & Distance $\mathrm{U}_{51}$ \\
\hline & & Number $\mathrm{U}_{52}$ \\
\hline & \multirow{5}{*}{ Environmental infrastructure $U_{6}$} & Security administration $\mathrm{U}_{61}$ \\
\hline & & SOS facilities $\mathrm{U}_{62}$ \\
\hline & & Delivery service $U_{63}$ \\
\hline & & Installation of lifts $\mathrm{U}_{64}$ \\
\hline & & Antiskid measures $\mathrm{U}_{65}$ \\
\hline
\end{tabular}

more indicators, so this paper used "three scale method" to reduce subjective error generated from the traditional method [5].

\section{Establishment of Indirect Judgment Matrix}

Firstly, using three scale method to calculate the comparison matrix:

$\boldsymbol{C}=\left(c_{i j}\right)_{n \times n}$

where,

$c_{i j}= \begin{cases}2 & \text { The } \mathrm{i} \text {-th element is more important than the } \mathrm{j} \text {-th element } \\ 1 & \text { The i-th element and the j-th element are equally important } \\ 0 & \text { The } \mathrm{j} \text {-th element is more important than the } \mathrm{i} \text {-th element }\end{cases}$

Then, calculate the sum value $\left(r_{i}=\sum_{j=1}^{n} c_{i j}\right.$ :) of each row. Elect the maximum $\left(r_{\max }\right)$ and the minimum $\left(r_{\min }\right)$, so comparative basis scaling $\left(d_{m}\right)$ can be calculated. Finally, indirect judgment matrix ( $\left.D=\left(d_{i j}\right)_{n \times n}\right)$ will be obtained.

$d_{i j}$ is expressed as:

$d_{i j}=\left\{\begin{array}{lc}\frac{!\left(r_{i}-r_{j}\right)}{\left(r_{\max }-r_{\min }\right)}\left(d_{m}-1\right)+1 & r_{i}-r_{j}>0 \\ ! ! & r_{i}=r_{j} \\ {\left[\frac{\frac{\left(r_{j}-r_{i}\right)}{\left(r_{\max }-r_{\min }\right)}\left(d_{m}-1\right)+1}{l^{-1}}\right]_{i}-r_{j}<0}\end{array}\right.$
2. The Weight of Level Element [6]

$\bar{A}=\left(\bar{a}_{i j}\right), \quad \bar{a}_{i j}=\frac{d_{i j}}{\sum d_{k j}}$. Calculate the sum value of each row of $\overline{\boldsymbol{A}}$, and $\bar{M}_{i}=\sum \bar{a}_{i j}$. Level elements feature vector will be obtained after standardization of $\overline{\boldsymbol{M}}$. The weight of level element:

$\boldsymbol{W}=\left[\omega_{1}, \omega_{2}, \cdots, \omega_{n}\right]$

where,

$\omega_{i}=\frac{\bar{M}_{i}}{\sum \bar{M}_{i}}$

3. Consistency Test

Consistency index:

$C I=\frac{\lambda_{\max }-n}{n-1}$

where,

$\lambda_{\max }=\sum_{i=1}^{n} \frac{(\boldsymbol{D W})_{i}}{n \omega_{i}}$

If $C I=0$, it shows the judgment matrix is consistent completely, or consistency ratio ( $C R=\frac{C I}{R I}$ ) should be calculated. If $C R<0.1$, the result is acceptable.

\section{ESTABLISHMENT OF FUZZY INTEGRATED EVALUATION SYSTEM}

\subsection{Establish Factors Set and Weight Set}

The main factors set: 
Table 2. The corresponding remark grade.

\begin{tabular}{|c|c|c|c|c|c|}
\hline Interval & $\mathbf{( 0 . 8 , 1 . 0 ]}$ & $\mathbf{( 0 . 6 , 0 . 8}]$ & $\mathbf{( 0 . 4 , 0 . 6 ]}$ & $\mathbf{( 0 . 2 , 0 . 4 ]}$ & $\mathbf{( 0 , 0 . 2}]$ \\
\hline \hline Conclusion & Excellent & Good & Medium & Pass & Fail \\
\hline
\end{tabular}

Table 3. Calculation criterion of evaluation value.

\begin{tabular}{|c|c|c|}
\hline Index & Evaluation Value & The Original Data Points and Fitting Curve \\
\hline Housing price & $Z_{1}=1-\frac{\left(x_{1}-\Delta\right)}{500} \times 0.1 \quad\left(0 \leq Z_{1} \leq 1\right)$ & $\begin{array}{c}\text { Remark: } x_{1} \text { represents residential real price, } \triangle \text { represents the ideal unit for residential } \\
\text { consumers. }\end{array}$ \\
\hline Traffic & $\begin{array}{c}\mathrm{Z}_{2}=\sum_{\mathrm{j}=1}^{\mathrm{k}} \mathrm{n}_{2 \mathrm{j}} \times \mathrm{y}_{2 \mathrm{j}} \\
Z_{2}=\sum_{j=}^{k} n_{2 j} \times y_{2 j}\left(\begin{array}{l}0 \leq Z_{2} \leq 1 \\
x_{2 j} \leq 800\end{array}\right)\end{array}$ & $\begin{array}{l}\quad x_{2}=[100: 100: 800] \\
y_{2}=[0.3,0.24,0.19,0.15,0.11,0.07,0.05,0.02] \\
y_{2}=-1.3 \times 10^{-10} x_{2}^{3}+4.4 \times 10^{-7} x_{2}^{2}-0.0007 x_{2} \\
+0.36\end{array}$ \\
\hline $\begin{array}{l}\text { Leisure and enter- } \\
\text { tainment }\end{array}$ & $\begin{array}{l}Z_{3}=\sum_{j=1}^{k} n_{3 j} \times y_{3 j} \\
\left(\begin{array}{l}0 \leq Z_{3} \leq 1 \\
x_{3 j} \leq 3500\end{array}\right)\end{array}$ & $\begin{array}{c}x_{3}=[600: 400: 3400] \\
y_{3}=[1,0.93,0.87,0.73,0.59,0.48,0.29,0.17] \begin{array}{l}y_{3}=2.1 \times 10^{-11} x_{3}{ }^{3}-1.7 \times 10^{-7} x_{3}{ }^{2}+0.0001 x_{3} \\
+0.99\end{array}\end{array}$ \\
\hline $\begin{array}{l}\text { Large shopping } \\
\text { centers }\end{array}$ & $\begin{array}{c}\mathrm{Z}_{4}=\sum_{\mathrm{j}=1}^{\mathrm{k}} \mathrm{n}_{4 \mathrm{j}} \times \mathrm{y}_{4 \mathrm{j}} \\
Z=\sum_{j=1}^{k} n_{j} \times y_{j} \\
\left(\begin{array}{l}0 \leq Z_{4} \leq 1 \\
x_{4 j} \leq 4000\end{array}\right)\end{array}$ & $\begin{array}{c}x_{4}=[500: 500: 4000] \\
y_{4}=[1,0.92,0.81,0.69,0.54,0.43,0.25,0.17] \\
y_{4}=5 \times 10^{-15} x_{4}{ }^{4}-3.2 \times 10^{-11} x_{4}{ }^{3}+3.5 \times 10^{-8} x_{4}{ }^{2} \\
-0.00018 x_{4}+1.1\end{array}$ \\
\hline $\begin{array}{l}\text { Comprehensive } \\
\text { hospital }\end{array}$ & $\begin{array}{r}Z=\sum_{j=1}^{k} n_{j} \times y_{j} \\
\left(\begin{array}{l}0 \leq Z_{5} \leq 1 \\
x_{5 j} \leq 5000\end{array}\right) \\
\left(0 \leq \mathrm{Z}_{5} \leq 1, \mathrm{X}_{5 \mathrm{j}} \leq 5000\right)\end{array}$ & $\begin{array}{c}x_{5}=[800: 600: 5000] \\
y_{5}=[1,0.97,0.84,0.72,0.63,0.05,0.40,0.24] \\
y_{5}=-7.5 \times 10^{-15} x_{5}^{4}+8.9 \times 10^{-11} x_{5}{ }^{3}-3.7 \times 10^{-7} x_{5}^{2} \\
+0.00045 x_{5}+0.84\end{array}$ \\
\hline $\begin{array}{l}\text { Environmental infra- } \\
\text { structure }\end{array}$ & $\begin{array}{l}Z_{6}=W_{6} \cdot \underline{R}_{6} \\
\left(0 \leq Z_{6} \leq 1\right)\end{array}$ & $\begin{array}{c}\text { Remark: } Z_{6} \text { was calculated by traditional fuzzy analysis on the basis of the actual situa- } \\
\text { tion of the five sub-indicators and the weight set. }\end{array}$ \\
\hline
\end{tabular}

$U=\left\{U_{1}, U_{2}, U_{3}, \cdots, U_{n}\right\}(i=1,2,3, \cdots, n)$

Sub-factors set:

$U_{i}=\left\{U_{i 1}, U_{i 2}, U_{i 3}, \cdots, U_{i m}\right\}$ !

Then, we get the main weight set:

$\boldsymbol{W}=\left(\omega_{1}, \omega_{2}, \cdots, \omega_{n}\right)\left(\sum_{i=1}^{n} \omega_{i}=1\right)$

and sub- weight set:

$\boldsymbol{W}_{i}=\left(\omega_{i 1}, \omega_{i 2}, \cdots, \omega_{i m}\right)\left(\sum_{j=1}^{m} \omega_{i j}=1\right)$
Each evaluation index is divided into 5 grades, evaluation set: $V=\left(V_{1}, V_{2}, V_{3}, V_{4}, V_{5}\right)=($ Excellent, Good,Medium, Pass, Fail $)$, its score set is $H=(1.0,0.8,0.6,0.4,0.2)$.

\subsection{Evaluation Value $Z_{k}$}

If a certain place exists an indicator, $n_{i j}$ represents the number of this indicator, $x_{i j}$ represents the distance between the house and this indicator. $y_{i}$ represents the fitting function obtained by MATLAB on the basis of survey data. 


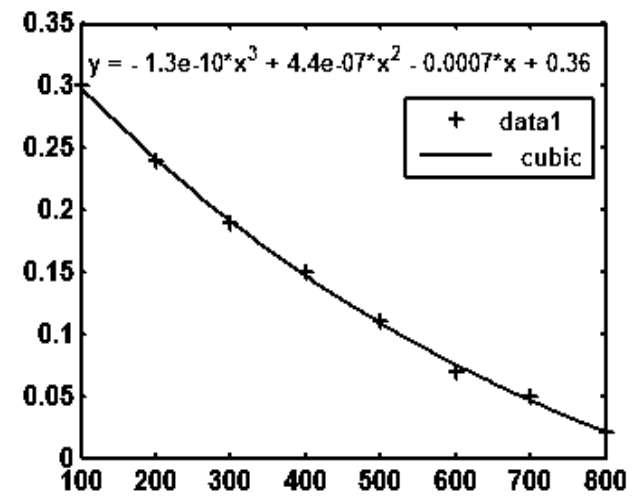

Fig. (1). Fitting curve of traffic.

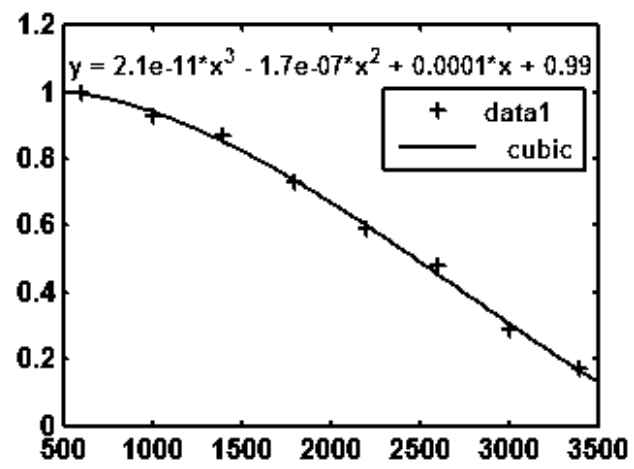

Fig. (2). Fitting curve of leisure and entertainment.

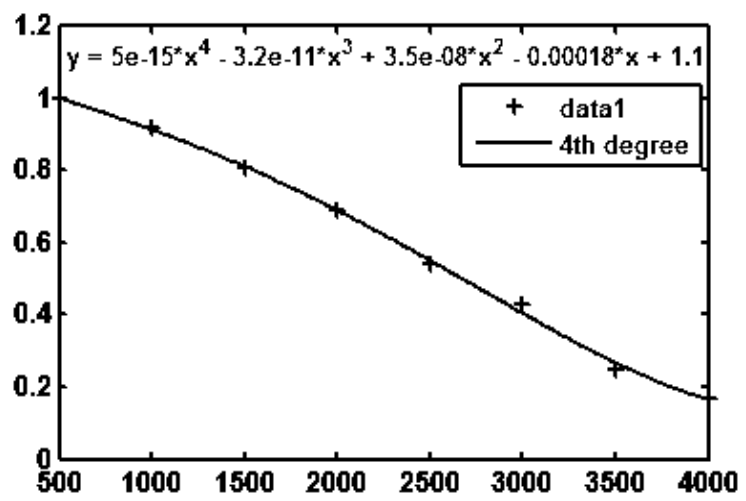

Fig. (3). Fitting curve of large shopping centers.

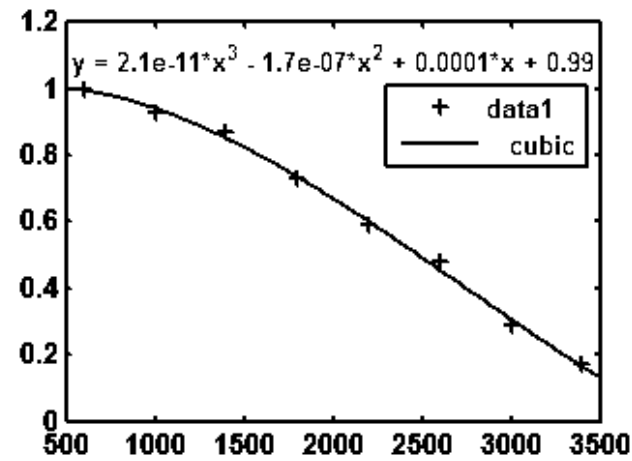

Fig. (4). Fitting curve of comprehensive hospital.

\subsection{Fuzzy Synthetical Judgment of $U$}

Comprehensive evaluation matrix:

$\underline{\boldsymbol{Z}}_{\text {total }}=\left(Z_{1}, Z_{2}, Z_{3}, Z_{4}, Z_{5}, Z_{6}\right)^{T}$
The result of evaluation system is its comprehensive score:

$h=\underline{\boldsymbol{B}}=\boldsymbol{W} \cdot \underline{\boldsymbol{Z}}_{\text {total }}$

\section{CASE ANALYSIS}

Using the sum product method to calculate the weights of evaluation system.

5.1. $\boldsymbol{U}-\boldsymbol{U}_{i}$

$$
\begin{gathered}
\boldsymbol{D}=\left[\begin{array}{cccccc}
1 & 1 & 5 & 3 & 3 & 5 \\
1 & 1 & 5 & 3 & 3 & 5 \\
\frac{1}{5} & \frac{1}{5} & 1 & \frac{1}{3} & \frac{1}{3} & 1 \\
\frac{1}{3} & \frac{1}{3} & 3 & 1 & 1 & 3 \\
\frac{1}{3} & \frac{1}{3} & 3 & 1 & 1 & 3 \\
\frac{1}{5} & \frac{1}{5} & 1 & \frac{1}{3} & \frac{1}{3} & 1
\end{array}\right] \\
\overline{\boldsymbol{A}}=\frac{d_{i j}}{\sum d_{k j}}=\left[\begin{array}{llllllll}
0.32609 & 0.32609 & 0.27778 & 0.34615 & 0.34615 & 0.27778 \\
0.32609 & 0.32609 & 0.27778 & 0.34615 & 0.34615 & 0.27778 \\
0.06522 & 0.06522 & 0.05556 & 0.03846 & 0.03846 & 0.05556 \\
0.10869 & 0.10869 & 0.16667 & 0.11539 & 0.11539 & 0.16667 \\
0.10869 & 0.10869 & 0.16667 & 0.11539 & 0.11539 & 0.16667 \\
0.06522 & 0.06522 & 0.05556 & 0.03846 & 0.03846 & 0.05556
\end{array}\right] \\
\overline{\boldsymbol{M}}=\sum \bar{a}_{i j}=\left[\begin{array}{lllllll}
1.90004 & 1.90004 & 0.31848 & 0.78150 & 0.78150 & 0.31848
\end{array}\right]^{0}
\end{gathered}
$$
$\omega_{i}=\frac{\bar{M}_{i}}{\sum \bar{M}_{i}}$.

The weight set:

$$
\begin{aligned}
& \boldsymbol{W}=\left[\omega_{1}, \omega_{2}, \cdots, \omega_{n}\right] \\
& =(0.3167,0.3167,0.0531,0.1302,0.1302,0.0531) \\
& \lambda_{\max }=\sum_{i=1}^{n} \frac{(\boldsymbol{D} \boldsymbol{W})_{i}}{n \omega_{i}}=6.0775, C I=\frac{\lambda_{\text {max }}-n}{n-1}=0.0155
\end{aligned}
$$

$C R=\frac{C I}{R I}=\frac{0.0155}{1.24}=0.0125<0.1$, so it has a satisfactory consistency.

5.2. $\boldsymbol{U}_{6}-\boldsymbol{U}_{6 j}$

$$
\boldsymbol{D}=\left[\begin{array}{ccccc}
1 & 1 & \frac{5}{2} & 4 & 4 \\
1 & 1 & \frac{5}{2} & 4 & 4 \\
\frac{2}{5} & \frac{2}{5} & 1 & \frac{5}{2} & \frac{5}{2} \\
\frac{1}{4} & \frac{1}{4} & \frac{2}{5} & 1 & 1 \\
\frac{1}{4} & \frac{1}{4} & \frac{2}{5} & 1 & 1
\end{array}\right]
$$




$$
\begin{gathered}
\overline{\boldsymbol{A}}=\frac{d_{i j}}{\sum d_{k j}}=\left[\begin{array}{lllll}
0.34483 & 0.34483 & 0.36765 & 0.32000 & 0.32000 \\
0.34483 & 0.34483 & 0.36765 & 0.32000 & 0.32000 \\
0.13793 & 0.13793 & 0.14706 & 0.20000 & 0.20000 \\
0.08620 & 0.08620 & 0.05882 & 0.08000 & 0.08000 \\
0.08620 & 0.08620 & 0.05882 & 0.08000 & 0.08000
\end{array}\right] \\
\overline{\boldsymbol{M}}=\sum \bar{a}_{i j}=\left[\begin{array}{lllll}
1.69731 & 1.69731 & 0.82292 & 0.31122 & 0.31122
\end{array}\right]^{T} \\
\omega_{i}=\frac{\bar{M}_{i}}{\sum \bar{M}_{i}} .
\end{gathered}
$$

The weight set: $\boldsymbol{W}_{6}=\left[\omega_{1}, \omega_{2}, \cdots, \omega_{n}\right]=(0.3507,0.3507$, $0.1706,0.064,0.064)$

$$
\lambda_{\max }=\sum_{i=1}^{n} \frac{(\boldsymbol{D} \boldsymbol{W})_{i}}{n \omega_{i}}=5.079, C I=\frac{\lambda_{\max }-n}{n-1}=0.0198 C R=
$$

$\frac{C I}{R I}=\frac{0.0198}{1.12}=0.0177<0.1$, so it has a satisfactory consistency.

If an older consumer want to buy a house, his expected price is 5000 yuan $/ \mathrm{m}^{2}$, the real price of a residence is 6000 yuan $/ \mathrm{m}^{2}$. Within 800 meters away from the house, there are three buses available at the distance of 200 meters and one bus available at the distance of 350 meters. Within 3500 meters away from the house, there is only one recreation area available at the distance of 1300 meters. Within 4000 meters away from the house, there is one large shopping centers at the distance of 2000 meters, another is at the distance of 3500 meters. Within 5000 meters away from the house, there is one comprehensive hospital at the distance of 2800 meters. There is no elevator in this community, but with emergency facilities, delivery business and anti-skid facility.

According to Table 3, we get:

$$
\begin{aligned}
& Z_{1}=1-\frac{(6000-5000)}{500} \times 0.1=0.8 \\
& Z_{2}=\left(-1.3 \times 10^{-10} \times 200^{3}+4.4 \times 10^{-7} \times 200^{2}-0.0007 \times 200+0.36\right) \times 3 \\
& +\left(-1.3 \times 10^{-10} \times 350^{3}+4.4 \times 10^{-7} \times 350^{2}-0.0007 \times 350+0.36\right) \times 1 \\
& =0.8730 \\
& Z_{3}=\left(2.1 \times 10^{-11} \times 1300^{3}-1.7 \times 10^{-7} \times 1300^{2}+0.0001 \times 1300+0.99\right) \times 1 \\
& =0.8788 \\
& Z_{4}=\left(5 \times 10^{-15} \times 2000^{4}-3.2 \times 10^{-11} \times 2000^{3}+3.5 \times 10^{-8} \times 2000^{2}\right. \\
& -0.00018 \times 2000+1.1) \times 1+\left(5 \times 10^{-15} \times 3500^{4}-3.2 \times 10^{-11} \times 3500^{3}\right. \\
& \left.+3.5 \times 10^{-8} \times 3500^{2}-0.00018 \times 3500+1.1\right) \times 1=0.9811 \\
& Z_{5}=\left(-7.5 \times 10^{-15} \times 2800^{4}+8.9 \times 10^{-11} \times 2800^{3}-3.7 \times 10^{-7} \times 2800^{2}\right. \\
& +0.00045 \times 2800+0.84) \times 1=0.6919
\end{aligned}
$$

$$
\begin{aligned}
& Z_{6}=\boldsymbol{W}_{6} \cdot \underline{\boldsymbol{R}}_{6} \\
& =(0.3507,0.3507,0.1706,0.064,0.064) \cdot\left[\begin{array}{c}
0.73 \\
0.82 \\
0.76 \\
0 \\
0.88
\end{array}\right]=0.7296
\end{aligned}
$$

Then, we get : $\underline{\boldsymbol{Z}}_{\text {total }}=(0.8000,0.8730,0.8788,0.9811$, $0.6919,0.7296)^{T}$.

Make the fuzzy synthetical judgment of $U, h$ will be calculated.

$$
\begin{aligned}
& h=\underline{\boldsymbol{B}}=\boldsymbol{W} \cdot \underline{\boldsymbol{Z}}_{\text {total }} \\
& =(0.3167,0.3167,0.0531,0.1302,0.1302,0.0531) \cdot\left[\begin{array}{l}
0.8000 \\
0.8730 \\
0.8788 \\
0.9811 \\
0.6919 \\
0.7296
\end{array}\right]
\end{aligned}
$$

$=0.8331$

So the comprehensive score of this elderly housing is 0.8331 , remark grade is "excellent". Similarly, other alternative systems' comprehensive scores can be calculated in the same way. Through a rational sort of those scores, a more appropriate housing will be selected.

\section{CONCLUSION}

With the increase of aging population, how to solve the question of senior's inhabitation is very exigent. This paper concluded a method to calculate evaluation value of the evaluation index system of elderly housing choice on the basis of FAHP. This method has strong operability and wide scope of application. Case analysis shows that the improved FAHP method has great practical value to solve the question of senior's inhabitation. Of course, due to regional differences, different factors will be considered in different regions, the specific differences can be improved on the basis of this paper.

\section{CONFLICT OF INTEREST}

The authors confirm that this article content has no conflict of interest.

\section{ACKNOWLEDGEMENTS}

Declared none.

\section{REFERENCES}

[1] The National Committee on Aging. Research report that predicts the development trend of Chinese aging population, [E book] Available From: Safari e-book. [Accessed: Feb. 12, 2007].

[2] Zhao Yang, "Problems and suggestions of elderly residential development in our country," Construction Economy, vol. 2, pp. 8890, 2008. 
[3] Jiang Qiyuan, "Some Issues in the Applications for the Analytic Hierarchy Process," Mathematics in Practice and Theory, vol. 23, pp. 156-168, 2013.

[4] Satty T L, "Vargas L G. Uncertainty and rank order in the analytic hierarchy process," European Journal of Operational Research, vol. 1, pp. 107-117, 1987.
[5] Tang Youchun, Fan Junhui, Method and application of systems engineering, CA: Beijing, Tsinghua University press, 2011, pp. 6567.

[6] Yu Haihui, "Constructs and measures of the soft power of enterprises based on the fuzzy hierarchy evaluation index," Statistics \& Decision, vol. 1, pp. 43-45, 2014.

Received: June 10, 2015

Revised: July 29, 2015

Accepted: August 15,2015

(C) Hongjun et al.; Licensee Bentham Open.

This is an open access article licensed under the terms of the (https://creativecommons $\mathrm{rg} /$ licenses/by/4.0/legalcode), which permits unrestricted, noncommercial use, distribution and reproduction in any medium, provided the work is properly cited. 